INTRODUCCIÓN

\title{
APUNTES PARA UNA MODELIZACIÓN: LA REGIÓN RÍO DE LA PLATA DURANTE SU PERTENENCIA A LA MONARQUÍA HISPÁNICA Y EN EL PROCESO DE FORMACIÓN DEL ESTADO-NACIÓN ARGENTINO*
}

\author{
INTRODUCTION \\ NOTES FOR A MODEL: THE RÍO DE LA PLATA REGION DURING ITS \\ BELONGING IN THE HISPANIC MONARCHY, INTO THE ARGENTINE \\ NATION-STATE FORMATION PROCESS
}

Fernando Jumar (dir.)

CONICET / Inst. de Est. Históricos (UNTreF) Academia Nacional de la Historia https://orcid.org/0000-0002-5926-5770

fjumar@conicet.gov.ar

\section{Recibido: febrero 23 de 2019 - Aceptado: abril 15 de 2019}

El conjunto de preguntas que guían mis investigaciones se relaciona con el análisis de la formación del Estado nacional argentino en una duración que incluye los Tiempos Modernos, estudiados a su vez como algo en sí y no ya como una larga transición. Ante la variada oferta de perspectivas teóricas existentes para enmarcar la profesión y aunque intento contemplar todas las variables de lo social, privilegio los enfoques que tienen en cuenta los factores económicos, vistos como la puesta al desnudo de las intenciones profundas de los sujetos históricos en acción. Lo económico, no es necesario argumentar demasiado, revela lo que las personas queremos, pero más usualmente lo que tenemos que hacer para subsistir, y lo que hacen aquellas que una vez que superaron ese escalón se puedan lanzar a la aventura de alcanzar metas mayores, que por lo que vemos los historiadores, siempre se logran por el establecimiento de relaciones de dominación de unos sobre otros. Relaciones exteriorizadas en cada tiempo histórico en entramados institucionales e ideológicos que logran, como por arte del encanto, que los dominados durante largos períodos de tiempo aceptemos y agradezcamos la dominación, y que inclusive, llegado el caso, hagamos el sacrificio de la vida, ayer en nombre de Dios y el Rey, hoy en el de la Patria y mañana, cuando haya cambiado una vez más el sistema de dominación, como vienen cambiando desde que tenemos noticias, vaya a saber uno en nombre de qué. 
En primer objetivo de investigación fue abordar el estudio de ambas bandas del Río de la Plata de modo integral durante la sujeción al dominio español ya que abría posibilidades explicativas y sumaba, desde otro ángulo, respuestas a viejas preguntas. Como pueden ser las relacionadas con las características de la economía rioplatense durante el Antiguo Régimen, lo que estaba en juego allí para propios y extraños, o la relación entre lo económico y los intereses que logran expresarse políticamente dentro del entramado de poderes policéntricos y parcialmente yuxtapuestos que componían la Monarquía Hispánica. Ello derivó, sin querer, en el establecimiento de un punto de partida levemente distinto al más usualmente utilizado en las explicaciones provistas para el proceso en que toman forma juntos tras 1810, tanto el Estado argentino como la Nación que lo sustenta.

Dedicaré parte del ensayo a la presentación del punto de vista que me lleva pensar que la historia de la formación, esplendor, crisis y disolución de un complejo portuario que articuló ambas bandas del río de la Plata en una única región ofrece claves interesantes para acercarse al problema de la formación del Estado-nación, al tiempo que muestra de otro modo la sociedad regional durante los Tiempos Modernos. En segundo lugar, sintetizaré el estado actual de lo que sabemos o creemos saber junto a los investigadores a quienes orienté u oriento, engarzándolo con las hipótesis que proyectan ideas hasta mediados del siglo XIX.

De modo que voy a exponer algo de conocimiento consolidado, emergente del reordenamiento de informaciones recogidas de la bibliografía y algunas generadas por nosotros, lo que deriva en muchas hipótesis de trabajo con las que se intenta estilizar una modelización explicativa.

El punto de vista con que abordo el problema se construyó bajo la influencia de la historia económico-social à la française, originalmente sin mayores pretensiones que la de encarar el estudio del comercio ultramarino rioplatense entre fines del siglo XVII y la creación del Virreinato del Río de la Plata, teniendo presente una recomendación de Samuel Amaral sobre que si me dedicaba el tema, era necesario incorporar más niveles de análisis en torno a la historia de Colonia del Sacramento en manos portuguesas. ${ }^{1}$

\footnotetext{
1 Fernando Jumar, "Les rioplatenses et le Río de la Plata. Les modalités du commerce rioplatense et les groupes locaux, 1680-1777”, Mémoire d’Études Appofondies (=maestría), inédita, École des Hautes Études en Sciences Sociales, 1988; "Le commerce atlantique au Río de la Plata, 1680-1778", Tesis de Doctorado, École des Hautes Études en Sciences Sociales, 2000. Hay una versión publicada: Le commerce atlantique au Río de la Plata, 1680-1778, 2da. ed. Aumentada, $1^{\text {a }}$ ed. electrónica, La Plata, UNLP, 2010, http://www.memoria.fahce.unlp.edu.ar/tesis/te.364/te.364.pdf (consulta 10/02/2019).
} 
Ciertamente, el comercio ultramarino rioplatense y Colonia del Sacramento habían recibido atención, mucha atención. Desde el nacimiento mismo de la historiografía científica argentina ya que, se sabe, Bartolomé Mitre, en su Historia de Belgrano... les brinda papeles destacados en las explicaciones que aporta al esclarecimiento del proceso que llevó a la Revolución de Mayo. ${ }^{2}$

Mitre establece y toda la historiografía posterior recoge, detalle más, detalle menos, el relato de base que aún domina sobre los cambios profundos que colaboran con la llegada de la independencia. Coloca el eje de lo que llevó a los americanos al cambio de mentalidad, que a su vez los llevó a la independencia, en una búsqueda innata por las vías que condujeran al liberalismo, desde mucho antes de que comenzara a tomar forma como cuerpo de doctrina. Por ejemplo, los cabildos, son en su historia organismos en los que se expresaba una “democracia genial”, siendo lo genial no una adjetivación positiva, sino la definición de una característica del "genio", de la "identidad primaria" diríamos hoy. El contrabando es entendido como justa desobediencia a una ley injusta, y conduce a una revolución económica que antecede y prepara la revolución política. Como se ve por los trabajos de Marta Poggi, es la "lucha por el libre comercio", que figuraba en nuestros manuales escolares como una de las "causas internas" de la Revolución. ${ }^{3}$ Colonia del Sacramento forma parte del proceso, es el nido de los contrabandistas en el siglo XVIII y, naturalmente, aparece en primer plano en la creación del Virreinato del Río de la Plata, hecho explicado como un momento de condensación de tendencias en un tiempo histórico que se acelera y en el que se redefinen, bajo el influjo de la coyuntura, un conjunto de variables que llevarán a buen término la larga gestación de una nación que tomará consciencia de sí misma, y al hacerlo, buscará su independencia.

La explicación en conjunto fue sometida a diversos análisis, inclusive fue reformulada y parcialmente rechazada por aportes posteriores, basados en otras metodologías y otros puntos de vista que el suyo. No es mi intención hacer el balance ahora, pero sí señalar que, en todas las derivaciones, por derecha, izquierda o centro, quedó intacta la preeminencia del comercio en el proceso y de sus actores en la vida local.

\footnotetext{
2 Bartolomé Mitre, Historia de Belgrano y de la Independencia Argentina, $4^{\mathrm{a}}$ ed. definitiva, 3 Tomos, Buenos Aires: Editorial Felix Lajouane, 1887. Se desarrollan los argumentos que dan centralidad al comercio legal e ilegal en el aludido proceso en los capítulos I a X del Tomo 1, pp. 1-346. V. Fernando Jumar, "Las 'reformas borbónicas' en la formación de la historiografía argentina”, en Historia del Caribe, Universidad del Atlántico, 11, 29, 2016, pp. 69-97.

3 Marta Mercedes Poggi, "Educar al soberano. Los libros escolares, lugares de memoria, 1880-1890". Tesis de Licenciatura inédita, Universidad Nacional de Tres de Febrero, 2004; "La consolidación y difusión de un mito fundacional. La Revolución de Mayo en los textos escolares, 1880-19052, Tesis de Maestría inédita, Universidad Nacional de Tres de Febrero, 2007. También ver: Isabel Paredes, "El Sesquicentenario de Mayo, la memoria y la acción editorial”, Universidad Nacional de Tres de Febrero, 2008.
} 
Al iniciar el posgrado en Francia en 1987 recibí de lleno el impacto de una renovación de los estudios sobre los Tiempos Modernos derivada de la puesta en relación de la historia, la memoria y la construcción y reproducción de las identidades nacionales.

Las preguntas que generaban esos estudios para la historia europea, aplicadas a la historia hispanoamericana, invitaban, entre otras cosas, a preguntarse a su vez si introducía distorsiones retrotraer en el tiempo para los análisis, a los tiempos de la dominación hispana del continente, la territorialidad de los estados-nación que algún día comenzaron a ser. En ello encontré ecos de propuestas hechas por Carlos Sempat Assadourian antes de que se lo preguntaran los modernistas de los años 1980 y que están en la base de las reflexiones que lo llevaron a formular su espacio peruano en $1972 .{ }^{4}$

Pero las preguntas de los modernistas también invitaban a interrogarse sobre el marco teórico general con que se aborda la explicación del lugar de América en la Monarquía Hispánica y las características del sistema de dominación, preguntas que de inmediato entendí ya que en la carrera de grado había sido acercado a las respuestas que se daban a través de los estudios relacionados con el derecho indiano. Pero, y si a pesar de lo que sostenía la gran mayoría de la historiografía, nacional e internacional, Las Indias no eran colonias, ¿no eran colonias por los motivos dados por Ricardo Levene ${ }^{5}$ o la explicación es otra? Y si no eran colonias, ¿̇qué eran? Y, además, ¿de qué hablamos cuando usamos 'colonia' como categoría analítica?

Felizmente, en la actualidad hay densas redes de especialistas en los tiempos modernos del mundo hispánico que abordan la cuestión y contamos con diversas respuestas

\footnotetext{
4 "Los límites geográficos impuestos a nuestro campo de observación requieren una breve explicación. Hemos esquivado el vicio tan frecuente de aplicar al tiempo colonial la noción moderna de espacio nacional que corresponde ciertamente a otra circunstancia histórica. El uso de esta arbitraria noción de espacio lleva a confusiones notables. Es que al levantar vallados y parcelar equívocamente los espacios reales de la historia colonial, los fenómenos económicos se vuelven ininteligibles a fuerza de ser circunscritos a extensiones geográficas que resultan inadecuadas para aprehenderlos en su totalidad. Recordemos a manera de simple ejemplo una forma de desvirtuación: en los análisis sobre la economía colonial se transforman en variables y factores externos aquellos que única y cabalmente son variables y factores internos. Teniendo en cuenta la trascendencia que conceden los interesados en los problemas del desarrollo a la distinción entre externo e interno, disipar la confusión no implica un amanerado cambio de palabras sino una precisión fundamental para detectar correctamente los procesos concretos." Carlos Sempat Assadourian, "Integración y desintegración regional en el espacio colonial. Un enfoque histórico", en EURE, Revista Latinoamericana de Estudios Urbanos Regionales, 2, 4, 1972, pp. 11-23, p. 11. Cursivas del original. Estas ideas se desarrollan in extenso en: Carlos Sempat Assadourian, "Sobre un elemento de la economía colonial: producción y circulación de mercancías en el interior de un conjunto regional", EURE. Revista Latinoamericana de Estudios Urbanos Regionales, 3, 8, 1973 , pp. 135-181, dentro de este artículo se puede consultar: "1. Consideración primera", pp. 135-138.

5 Ricardo Levene, Las Indias no eran colonias, $1^{\mathrm{a}}$ ed. en 1951, $3^{\text {a }}$ edición, Colección Austral \# 1060, Madrid, Espasa Calpe, 1973.
} 
en formación. ${ }^{6}$

Pero en su momento, al leer y re-leer la bibliografía desde las preguntas y la mirada que se estaba conformando a partir de los interrogantes hechos sobre los macro-relatos existentes, noté que en los estudios sobre el comercio, tanto la historiografía argentina como la uruguaya habían hecho del Río de la Plata una frontera internacional desde que llegó Solís (1516) , lo que llevaba a separar antes de tiempo la historia de sociedades que se construyeron juntas. También, que Colonia del Sacramento era analizada principalmente en función de su papel en las disputas inter-imperiales y como foco de contrabando, pero que poco y nada sabíamos de ella desde adentro y que, posiblemente, fuese algo más que un nido de contrabandistas, como pude demostrar primero y sobre todo refinar desde que se inició la colaboración con Isabel Paredes para entender qué estaba realmente en juego allí para la sociedad local.

Así, la primera pregunta concreta que me hice fue desde cuándo el río de la Plata es realmente una frontera internacional y sin proponérmelo, mi primer objetivo que era analizar el comercio rioplatense en el siglo XVIII pre-virreinal, se entroncó con el de la formación del Estado-nación.

Si el río podía no haber sido una frontera entre la Argentina "colonial” y el Uruguay “colonial”, entonces ¿era un vector de integración? Pero, ¿de qué?

Al volver los ojos a Río de la Plata y sus flujos mercantiles, mirando el río como un factor de unión y no ya como una frontera entre Argentina y Uruguay, y pensando Colonia del Sacramento como parte de la trama interna de la vida local, vi aparecer ante mis ojos lo que llamé el complejo portuario rioplatense. De paso, el río, se había convertido en mi Méditerranée... No tendría que abordar la historia de cada una de sus bandas por separado en tiempos de la sujeción a las coronas ibéricas, sino que se trataba de una sola historia, la de la región Río de la Plata en los Tiempos Modernos.

Para la búsqueda de los contornos y características generales de lo que podría ser

\footnotetext{
6 V. Fernando Jumar, "El mundo hispánico durante el Antiguo Régimen desde los circuitos mercantiles y el espacio económico rioplatense” en Historia Crítica, 70, Bogotá, 2018, pp. 23-44.

7 Estos aspectos se abordan en: Fernando Jumar, "Colonia del Sacramento, la bibliografía y la creación de las memorias nacionales”, en Alejandra Mailhe; Emir Reitano (Comps.), Pensar Portugal. Reflexiones sobre el legado histórico y cultural del mundo luso en Sudamérica, La Plata, Facultad de Humanidades y Ciencias de la Educación, 2008 , pp. 41-75.
} 
esa región Río de la Plata, integradora de las dos bandas del río, a través de los textos de Carlos Sempat Assadourian relacionados con el espacio peruano, llegué a los trabajos del economista François Perroux de los años 1950 y sus propuestas en torno a los espacios económicos, que tanto éxito tuvieron en las políticas desarrollistas de los años 1960. ${ }^{8}$

Los espacios económicos de Perroux, ya no pensados con los fines a futuro con que fueron concebidos, sino convertidos en herramientas del análisis histórico, permiten formular modelos analíticos en los que la observación se realiza desde la circulación mercantil, con el objetivo de llegar al mundo de la producción y a la esfera de la organización institucional del sistema de dominación.

La reflexión teórica y metodológica emprendida a partir tanto de los trabajos de Assadourian como de Perroux llevan a que el primer paso sea definir, sobre la base de la determinación de lo que pueden ser circuitos de comercialización interna de proximidad, lo que se llaman espacios económicos homogéneos. También, detectar las actividades productivas que los estructuran hacia dentro y que los ponen en relación con otros espacios económicos a través de la circulación. Mi único agregado a este esquema es que, para completar el cuadro de las economías regionales y sus relaciones con otras, había que sumar a la consideración de la acción fiscalizadora de la Corona en tanto que depredadora, su papel como agente dinamizador, a través del gasto realizado para el sostenimiento de los aparatos burocráticos, judiciales, religiosos y militares del sistema de dominación.

El segundo paso es ver cómo esos espacios homogéneos se relacionan con otros. Cada espacio homogéneo tiene sus actividades productivas y puede ser que la actividad productiva dominante de uno de ellos genere efectos de arrastre sobre otros espacios, instalándose complementariedades que conducen a procesos de integración. Cuando eso pasa, surgen los espacios económicos polarizados, que según sea la intensidad de las relaciones que se tejan detrás de las económicas, pueden llegar a coadyuvar en la definición o redefinición de las relaciones políticas e institucionales entre ellos. Mi único aporte a este esquema es que si se considera este análisis para las sociedades americanas, hay que mantener la coherencia de la mirada y pensar en cómo se relacionan los espacios económicos americanos con los extra-americanos, y no relacionarlos con economías nacionales como se solía hacer. Una derivación es considerar los tratos ultramarinos americanos por dentro

8 V. Fernando Jumar, “El espacio colonial peruano en la historiografía sobre circulación mercantil”, en História Ecônomica \& História de Empresas, 17, 2, 2014, pp. 475-534. 
de la Carrera de Indias en tanto que fenómenos de circulación interna a gran distancia y no ya como comercio colonial.

En función de ese esquema, reordenando la información existente y aportando nueva, la región Río de la Plata durante los Tiempos Modernos estuvo constituida por territorios dominados efectivamente por los occidentales a ambas orillas de los ríos de la Plata y Uruguay, así como ríos Paraná y Uruguay arriba hasta Corrientes y los pueblos de las misiones jesuitas, pudiendo establecer unos límites aproximados hacia el interior de las tierras a través del tipo de tratos mercantiles que revelan la circulación entre la capital y consumidores y minoristas así como la provisión de insumos para la producción. Unos contornos cambiantes a lo largo del tiempo que llegan a una extensión e integración máximas en el período virreinal.

El mundo de la producción regional, analizado en función de este esquema ha sido abordado por Nicolás Biangardi. Observa que desde mediados del siglo XVIII la región se encontraba en un proceso de expansión territorial y crecimiento demográfico de manera que las fronteras se desplazaban, se incorporaban nuevas tierras para la explotación de la ganadería extensiva, el sector de producción agropecuaria mixta se expandía y la zona de servicios y transformación de productos del complejo portuario se ampliaba. El resultado de su investigación pone en evidencia los intereses vinculados a la explotación del ganado bovino en las dinámicas internas de la región. ${ }^{9}$

Por su parte, María Emilia Sandrín analiza el impacto sobre la población local de la dinamización económica que deriva de las demandas de bienes y servicios requeridos por el propio funcionamiento del complejo portuario rioplatense y por la corona española para el mantenimiento de su dominio en la región. Observa que los efectos de esas demandas se dejan sentir hasta en las capas más sumergidas de la sociedad rioplatense, ya que cuando no son los barcos del comercio son los de la corona o sus soldados los queponen en movimiento un abigarrado mundo de minúsculos actores económicos, entre los que se encuentran desde nuestros viejos conocidos de los actos escolares, la mazamorrera o el vendedor de velas, hasta otros menos famosos, como las bizcocheras o los oficios asociados al carenado y calafateado de las embarcaciones. ${ }^{10}$

${ }_{9}^{9}$ Nicolás Biangardi, "Poblamiento, crecimiento económico y poderes locales en un área de la región Río de la Plata. Maldonado, 1755-1814”, Tesis de Maestría inédita, Universidad Nacional de Tres de Febrero, 2012; "Expansión territorial, producción ganadera y relaciones de poder en la región Río de la Plata. Montevideo y Maldonado a fines del siglo XVIII”, Tesis de Doctorado inédita, Universidad Nacional de La Plata, 2015.

${ }^{10}$ María Emilia Sandrín, "El sitio a Colonia del Sacramento, la economía y su gente, 1735-1737”, Tesis de Maestría, Universidad Nacional de Tres de Febrero, 2011. Hay una versión impresa: Bizcocheras, lancheros y demás... Los estímulos económicos de la guerra para los sectores medios y bajos del complejo portuario rioplatense, 1735-1737, Saarbrücken, Editorial Académica Española, 2011. De la misma autora: "La demanda de bienes y servicios para la Corona y 
La región Río de la Plata que se propone, entonces, es un espacio acuático y térreo, articulado por un complejo portuario, sin solución de continuidad a la hora de analizar los campos de fuerzas económicas que lo integran hacia dentro o de seguir el rastro de las que lo vinculan con otros espacios económicos americanos y extra-americanos.

La historia del complejo portuario rioplatense brinda un eje de seguimiento tanto de la conformación de la región como de sus relaciones con otras. Esa historia comienza con la invasión europea de la región, ya que desde el comienzo se necesitó de la articulación de ambas orillas del río para el mantenimiento de los contactos con las costas del Brasil y con las rutas atlánticas ultramarinas. Al calor de los esfuerzos desplegados por diversos actores extra-americanos por apropiarse de partes crecientes del tesoro americano a través de Río de la Plata, esa puerta trasera de los complejos mineros suramericanos surge, se consolida y madura una sociedad hispano-criolla que, en el tiempo, cada vez más va a pesar en el juego de relaciones y llega a tener una voz propia que no pudo ser ignorada. ${ }^{11}$

En la periodización de la vida del complejo portuario rioplatense se proponen cuatro etapas. Una que llamo formativa, entre el inicio de la instalación de los europeos en la región a fines del siglo XVI y los tiempos de la Guerra de Sucesión a inicios del siglo XVIII. Le sigue un breve período que entiendo como de redefinición y condensación de tendencias, entre los años de la guerra y la ocupación española de la bahía de Montevideo formalizada en 1726. En tercer término, lo que considero la etapa de pleno funcionamiento, entre mediados de los años 1720 y la década de 1810 (con un importante cambio del lugar de Colonia desde 1777), que es seguida por la cuarta y última etapa, la de crisis y disolución, que acompaña a la de la región Río de la Plata tal como la entiendo, cuyo fin advierto claramente en tiempos de la guerra económica entre la Confederación Argentina y el Estado de Buenos Aires durante los años 1850.

Durante la etapa formativa, observo que las adaptaciones a las imposiciones geográficas y las dinámicas generales de la historia de la Monarquía Hispánica en sus relaciones con sus competidores se combinan con un proceso de asentamiento y maduración de una sociedad local en la que intervienen dos factores esenciales. En primer término, su condición de frontera de la Monarquía lo que derivó en inversiones crecientes para la

la navegación ultramarina en el complejo portuario rioplatense y la dinamización de la economía regional, 1680-1810”, Tesis de Doctorado inédita, Universidad Nacional de La Plata, 2016.

${ }^{11}$ P. e. Fernando Jumar, "El precio de la fidelidad. La Guerra de Sucesión en el Río de la Plata, los intereses locales y el campo Borbón”, en Annie Molinié; Alexandra Merle (Dirs.), L’Espagne et ses guerres. De la fin de la Reconquête aux guerres de l’Indépendance, París, Presses de l’Université Paris/Sorbonne, 2004, pp. 203-236. 
afirmación de su soberanía. En segundo término, su condición de pivote entre el Atlántico y los mercados del sur americano. En el proceso tuvo una alta incidencia la acumulación de experiencias en relación con el comercio a media y larga distancia, por tierra o por agua, que permitió que la región pudiera responder en cada tiempo a los múltiples requerimientos que realizaba a su sociedad la función mediadora.

En ese proceso de acumulación de experiencias mercantiles se observa cómo los porteños, únicos ocupantes occidentales del río hasta avanzado el siglo XVII, van incorporándose de modo progresivo en los flujos, con un orden de lo simple a lo complejo que bien podría haber sido diseñado por un ministro planificador. Por detrás, se ve una economía regional que encuentra, además de cómo asegurar el funcionamiento de la ruta, el modo de que su comarca sea algo más que un pivote entre mercados americanos y extra-americanos, a través de producciones que se suman a los intercambios por tierra o por agua y que junto a los servicios portuarios generan riqueza o simplemente una moderada prosperidad para el conjunto de la población.

En 1680-1683, la instalación de los portugueses en la banda norte, en uno de los puertos informales hasta entonces del complejo portuario, encuentra del otro lado una comunidad con intereses arraigados, capaz de ofrecer servicios de mediación con una vasta red de mercados americanos, y dispuesta también a proteger su economía productiva. En 1703, con la instalación en Buenos Aires de la factoría de la Compagnie de Guinée et de l'Assiento, los porteños aprenden la rama del comercio que les faltaba, la de esclavos a gran escala (para el contexto, por supuesto).

La Guerra de Sucesión tuvo uno de sus escenarios bélicos en Río de la Plata, lo que altera la vida del complejo portuario rioplatense, ya que se expulsa a los portugueses en 1705 (expulsión reclamada por los porteños desde los años 1690 porque los portugueses estaban descaradamente explotando sus ganados de la banda norte) y se corta la llegada de navíos del comercio por dentro de la Carrera de Indias hasta 1712, dominando el tráfico ultramarino el comercio francés.

Esa guerra desatada en 1701 y sus ecos rioplatenses abren una breve etapa de reacomodamientos que dura hasta la primera consolidación de las derivaciones de los tratados de paz, que ubico en la ocupación española de Montevideo entre 1724-1726.

Sin embargo, es en estos años de guerra y reacomodamientos, que la economía 
rioplatense conoce su primer gran momento de crecimiento concentrado en el tiempo.

Un único indicador: según cifras aportadas por Zacarías Moutoukias, en los 52 años que median entre 1648 y 1699 se exportaron de modo legal 173.370 cueros, una media anual de 3.334 unidades. ${ }^{12}$ Está el contrabando, lo sé. También las vaquerías que organizan los portugueses en los años 1690. De modo que fantaseemos y cuadrupliquemos la media de los embarques legales de esos 52 años, lo que da 13.336 unidades potencialmente exportadas por año. Ahora bien, en los 12 años que corren entre 1703 y 1714 se registró legalmente el embarque de 296.962 cueros a bordo de navíos españoles (la salida de los del comercio llegados en 1698, más otros de guerra y avisos llegados entre 1701 y 1704) y los de la Compagnie de Guinée et de l'Assiento. Sin aportar estimaciones para los embarques por el circuito portugués (hasta la expulsión de 1705), ni para los fraudes por dentro del sistema español o de la compañía esclavista francesa o las compras y matanzas de los navíos franceses del comercio directo, la media anual es de 24.747 cueros. ${ }^{13}$ Un boom económico, ya que se experimentó en 12 años 85\% de crecimiento en relación a la estimación muy fantasiosa para los 52 años previos de las exportaciones del principal bien producido a escala regional, recordando que en este análisis la región Río de la Plata no se limita a la campaña próxima a Buenos Aires sobre la banda sur del río.

La contrapartida. La intensificación de la presencia del comercio ultramarino en los circuitos interiores que se abrían desde el complejo portuario rioplatense, y con ella el punto de inflexión en la polarización hacia el Atlántico de varias economías regionales. Los rioplatenses ven pasar por su complejo portuario cada vez más millones de pesos que no les pertenecen, pero que por pasar, generan estímulos a su economía lanzados en todas direcciones, porque no hay que pensar solamente en los cueros exportados, sino también en cómo los navíos del comercio y las necesidades del rey dinamizaron otras esferas de la economía regional.

El fin de la guerra y las condiciones de los tratados de paz cambiaron a los franceses por los ingleses en 1714 para el tráfico esclavista y devolvieron a los portugueses a Río de la Plata en 1716. El intento expansivo de los portugueses con la ocupación de la bahía de Montevideo a fines de 1723 termina con la fundación española de San Felipe de Montevideo, y

${ }^{12}$ En función de las salidas de los navíos de registro en 1648, 1654, 1658, 166o, 1661, 1663, 1669, 1671, 1674-1676, 1681, 1685, 1693, 1699. Z. Moutoukias, "Le Río de la Plata et l'espace péruvien au XVII ${ }^{\mathrm{e}}$ siècle : commerce et contrebande par Buenos Aires”, Tesis de Doctorado, École des Hautes Études en Sciences Sociales, Francia, 1983, Tableau 8, pp. 134-137.

${ }^{12}$ F. Jumar, Le commerce atlantique, Tableau D, Annexe 3, Ch. 5, pp. 611-617. 
queda el escenario armado para un largo período en el que el complejo portuario rioplatense funcionó a pleno, sumando integrantes medianos y menores, y aun teniendo presente la ajetreada vida de Colonia del Sacramento en manos portuguesas o las tensiones crecientes entre porteños y montevideanos claramente visibles desde 1808.

A mediados de la década de 1720 se abre, entonces, la etapa de pleno funcionamiento del complejo portuario rioplatense, que dura hasta los años 1810. Ese complejo portuario en su imbricación más densa estuvo compuesto, en primer lugar, por la ciudad de Buenos Aires con sus malos y transitorios apostaderos navales, más las ciudades-puerto de Colonia del Sacramento (bajo dominio portugués) y Montevideo. En segundo lugar, se articulaban con esas ciudades un reducido conjunto de apostaderos secundarios, aptos para naves mayores y medianas, con mayor o menor control por parte de las autoridades (como Maldonado o la Ensenada de Barragán). También con control escaso o al menos sin dudas deficiente, había atracaderos transitados por naves medianas y menores, fundamentalmente destinados a articular zonas productivas del litoral con el estuario del río, como Las Conchas (la terminal de la ruta fluvial que llevaba a Paraguay), pero que también eran útiles para vincular por agua las zonas productivas de la región rioplatense con los puertos mayores, como Las Vacas. Finalmente, un indefinible número de desembarcaderos clandestinos o simplemente sin control, permitidos por las características de las orillas, aptos para los movimientos de pequeñas embarcaciones, útiles no sólo para los tratos ilícitos sino también para los movimientos de una población que iba y venía de una banda a otra del río con mucha asiduidad siguiendo, por ejemplo, las fuentes de trabajo. Así, el río, debido a la intensa circulación de embarcaciones de todo porte entre ambas orillas o entre puntos distantes de alguna de ellas, se convirtió en el vector de integración y articulación regional, y él mismo en uno de los espacios que la componían.

En el análisis de las relaciones entre los asentamientos mayores del complejo portuario rioplatense entre sí hasta 1777, es decir, Buenos Aires, Colonia del Sacramento y Montevideo, descubro una fuerte complementariedad en lo relacionado con el comercio, pero la paz se acaba cuando se trata de los intereses productivos. Observo que lo que está en disputa es la explotación del ganado y que el punto de equilibrio se alcanza mientras Colonia del Sacramento y Montevideo acepten el papel que les asignan los intereses porteños, exteriorizados durante largo tiempo a través de su Cabildo en las negociaciones con los gobernadores o directamente con la Corona.

Así, las cifras obtenidas hasta ahora, por la bibliografía o producidas en los equipos 
de investigación que dirigí, lo mismo que el seguimiento de la vida política regional, sugieren que hay que volver a mirar los intereses vinculados a la explotación del ganado bovino, ya que su participación en los flujos, a pesar de ser claramente minoritaria frente a los metales preciosos, es importante si se la analiza desde la perspectiva de la inclusión de las economías regionales en el comercio ultramarino.

¿Cuáles son esos intereses vinculados a la explotación del ganado? Los defino como empresarios ganaderos que asumirían diversas formas a través del tiempo: vecinos accioneros, hacendados, estancieros. Nicolás Biangardi prefiere llamarlos corambreros en el período virreinal. Ni estanciero ni comerciante, o ambas cosas a la vez, lo que definiría al empresario ganadero es que habría colocado en el centro de sus objetivos y estrategias la obtención de beneficios a partir de la ganadería, única rama de la actividad económica vinculada al Atlántico que podría aspirar a controlar. ${ }^{14}$

¿Qué reflejan los millones en metales preciosos salidos, legal o ilegalmente, por el complejo portuario rioplatense? Ciertamente, no reflejan la economía rioplatense de modo principal, sino el conjunto de operaciones mercantiles llevadas a cabo en el cada vez más extenso y denso haz de rutas que se abren desde el complejo portuario rioplatense hacia el interior americano. La participación de la economía regional en la conformación del total sólo podría conocerse tras despejar qué parte de los bienes aportados por los navíos fueron consumidos en la región y lo que deja esa circulación en la región, es decir, tras despejar la parte que le corresponde a la sociedad local por sus servicios de mediación, desde las comisiones de los comerciantes o los beneficios de los comerciantes locales que operan por cuenta propia (que eran los menos), hasta los jamones comprados por los despenseros de los navíos para los viajes de regreso. Pero en esencia, se trata de un comercio que opera a través de Río de la Plata, pero que no es de Río de la Plata, tanto por el origen mayoritario de los fondos invertidos como por el destino de los beneficios. Bajo su estímulo se articula un espacio económico rioplatense, que logra revertir desde comienzos del siglo XVIII la polarización previa en dirección de Lima, arrastrando bajo su influjo inclusive parte de Chile (el corregimiento de Cuyo) y, por decisiones políticas, también se busca que se polaricen, o se terminen de polarizar, el Alto Perú y Paraguay con su inclusión en el Virreinato del Río de la Plata en 1776.

${ }^{14}$ Un ejemplo de este tipo de actor lo da el caso de Antonio Obligado. V. Juan Ignacio Pieretti-Bravo, "De comerciante a Apoderado del Gremio de Hacendados. El ascenso político de Antonio Obligado, 1765-1793”, Tesis de Licenciatura inédita, Universidad Nacional de Tres de Febrero, 2018. 
Pero por debajo de los grandes flujos que desmonetizan las economías regionales, encontramos una de esas economías que aporta bienes en dirección del Atlántico que retienen en América al menos una parte de los metales preciosos, los derivados bovinos rioplatenses. En su momento, pude acceder a un conocimiento muy detallado de lo cargado en los navíos del circuito legal español y la participación de los actores en las transacciones gracias a una aplicación informática programada por Alejandro Zurdo. ${ }^{15}$ Los resultados mostraron que en el conjunto de los valores exportados entre 1714 y 1778 los cueros pesan poco, pero mucho más que el $2 \%$ que suman todas las otras producciones americanas que salen por el complejo portuario rioplatense hacia el Atlántico por dentro del circuito legal español, llegadas desde zonas tan remotas como Guayaquil, Moxos, la selva misionera o el Atlántico sur. Entre esos años, 1714 y 1778, los cueros representan alrededor de 10\% del total y hay estimaciones que sugieren que alcanzan 20\% durante el período virreinal. Así que es cierto, son poca cosa en el conjunto de valores salidos por el circuito legal español. La ponderación general al incluir los tratos ilegales tal vez no cambie mucho, ya que si bien salen muchos cueros por el circuito portugués (más que por el español hasta los años 1750-1760), también salen metales preciosos. Pero no es una cuestión de proporciones, sino de constatar que una sola de las economías regionales interviene en los flujos ultramarinos con un bien de producción propia que retiene en la región parte de la riqueza generada en otras y que ese poco, para la economía regional, es mucho. A lo que se suma el ganado en pie, bovino o mular, que sale en grandes cantidades hacia el interior español, pero también al portugués. Y se completa el cuadro de la economía regional con lo gastado por el comercio o por el rey para la satisfacción de sus necesidades de bienes y servicios.

En cuanto a los actores, esperaba validar la idea que propone que los comerciantes en todo tiempo controlaron la coordenada política local a través del Cabildo, pero cuando no encontré a los cabildantes entre los cargadores ultramarinos, primero me desorienté y luego pude ver que al menos hasta la creación del Virreinato del Río de la Plata, la corporación urbana habría trabajado para defender los intereses productivos locales.

¿Cómo se articula el comercio de importación de bienes del mercado mundial a través de complejo portuario rioplatense con la exportación de derivados bovinos? No hay demasiado misterio. La economía regional se dinamiza en la medida que haya navíos en el río que atender y bodegas disponibles para los cueros. No llegan navíos del comercio

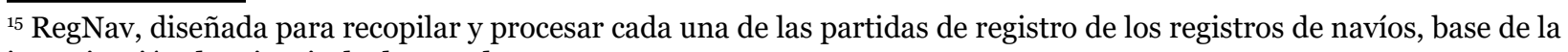
investigación de mi tesis de doctorado. 
en lastre para comprar cueros y aunque en expansión, el mercado rioplatense no puede absorber los bienes del mercado mundial que llegan al complejo portuario rioplatense. Como la enorme variedad de textiles puestos en circulación revelados por las notas de aduana.

Los rioplatenses tienen el mayor interés en que el comercio ultramarino se articule con los espacios interiores a través de su complejo portuario, para que una vez vaciadas las bodegas, puedan ser llenadas con sus cueros.

De la articulación entre los intereses locales y ultramarinos emerge un espacio económico polarizado, el espacio económico rioplatense. Un espacio económico que tal vez anticipe en un siglo el quiebre del espacio peruano tal como lo había propuesto Assadourian y que quedaría delimitado por el complejo portuario rioplatense, que se proyecta aguas arriba hacia el Paraguay (estudiado por Isabel Paredes primero ${ }^{16}$ y más tarde por Maximiliano Acebal ${ }^{17}$ ), y dos complejos portuarios secos, el mendocino-sanjuanino, estudiado por José Sovarzo ${ }^{18}$ y el salto-jujeño, percibido por ahora a través de la bibliografía, ya que todavía está buscando a su tesista. Todo articulado por una densa red de caminos de agua y tierra, animados por diversos tipos de empresarios transportistas, analizados por María Claudia Errecart ${ }^{19}$

Las complementariedades y contradicciones derivadas de la articulación de intereses definen la vida regional y la participación de sus minorías dirigentes en el juego de poderes de la Monarquía española, una monarquía que interviene en la vida regional en función de su primer interés, que es asegurar esa frontera caliente, con lo que se transforma en un muy activo agente dinamizador de la economía regional, gastando en la región

\footnotetext{
${ }^{16}$ Isabel Paredes, "El comercio de la subregión de Paraguay a fines del siglo XVIII", en XIII Jornadas Interescuelas/departamentos de Historia, Universidad Nacional del Comahue, San Carlos de Bariloche, 2009, http://cdsa.aacademica.org/ooo-008/610.pdf (consulta 10/02/2019); "La carrera del Paraguay a fines del siglo XVIII", en América Latina en la Historia Económica, 21, 1, 2014, pp. 66-91; "Los transportistas asuncenos de la Carrera del Paraguay y sus relaciones comerciales con Buenos Aires", en XIV Jornadas Interescuelas/Departamentos de Historia, Universidad Nacional de Cuyo, Mendoza, 2013, https://www.aacademica.org/ooo-010/264 (consulta 10/02/2019). ${ }^{17}$ Maximiliano Acebal, “Caminos del río Paraguay: Asunción y sus áreas de influencia económica, 1779-1783”, en 6tas. Jornadas de Historia Económica, Asociación Uruguaya de Historia Económica, Montevideo, 2-4 de diciembre de 2015. ${ }^{18}$ José Sovarzo, "La garganta del comercio interoceánico en la América del sur hispana. Mendoza y sus relaciones comerciales con Río de la Plata y los mercados del Pacífico Sur americano”, Tesis de Maestría inédita, Universidad Nacional de Tres de Febrero, 2017.

${ }^{19}$ Proyecto de posgrado en curso: "Caminos, transportes y transportistas en el espacio económico rioplatense", Universidad Nacional de Tres de Febrero.
} 
sumas de dinero recaudadas en otros espacios americanos y extra-americanos. ${ }^{20}$ En segundo término, interviene reorganizando la estructura administrativa general, creando un virreinato que le permita controlar mejor los flujos del espacio económico rioplatense transformándolo en uno de planificación al incluir entre sus mercados preferenciales Alto Perú y Paraguay, y buscando controlar los flujos del conjunto a través de la aduana porteña, que está siendo estudiada por María Evangelina Vaccani²1.

La crisis de la Monarquía española desatada a comienzos del siglo XIX, y la forma que asume localmente su resolución entre 1806 y 1820, quebró la unidad de la región Río de la Plata. Tras ella se quiebra la del complejo portuario en torno a la que se había articulado y se descalabra el espacio económico virreinal.

En 1777 la expulsión de los portugueses de la banda norte del río se dio en el contexto de una serie de reformas que apuntaban a expandir el absolutismo en el conjunto de los dominios europeos, americanos y asiáticos de la Monarquía Hispánica. Con la conquista de Colonia del Sacramento, el complejo portuario perdió en tanto que puerto mayor un componente que generaba equilibrios, siempre inestables, entre porteños y montevideanos, más en el momento en que Montevideo estaba llegando a su primera madurez. Como lo muestra Maximiliano Camarda para el complejo portuario rioplatense en el período virreinal, hay un incremento del comercio. ${ }^{22}$ Pero si las cifras se ponen en relación con las aportadas para el período 1714-1778, ese crecimiento no es tan abrupto como se pensaba, sino que continúa tendencias previas que se originan en las dos primeras décadas del siglo XVIII y se sostienen después al incorporar al análisis el circuito portugués. El salto de las curvas que contemplan sólo el comercio legal español tras 1778 se debe a la concentración forzada de la circulación por ese circuito, tras la toma de Colonia del Sacramento.

Sin embargo, el relativo éxito del avance del absolutismo en la región, que como explica Javier Kraselsky fue posibilitado por una alianza entre la corona y un actor local, los comerciantes de Buenos Aires, genera nuevas tensiones dentro de la región mediante

\footnotetext{
${ }^{20}$ Se enviaban desde la Corte sumas de dinero para cubrir al menos parte de los gastos generados por las expediciones militares. P. e. la expedición de Cevallos de 1776-1776 aportó 800.0oo pesos. Sobre los aspectos económicos de esta expedición ver: Rubén Da-río Serrato Higuera, "Las armas del dinero: Mecanismos de financiación y administración en la segunda expedición de Pedro de Cevallos al Río de la Plata, 1777”, Tesis de Maes-tría, Universidad Nacional de Tres de Febrero, 2017.

${ }^{21}$ Proyecto de posgrado en curso: "La Real Aduana de Buenos Aires en el período virrei-nal (1778-1796)”, Universidad Nacional de Tres de Febrero.

${ }^{22}$ Maximiliano Camarda, "Comercio Ultramarino de cueros del complejo portuario rioplatense. 1779- 1799". Universidad Nacional de Mar del Plata, Tesis de Maestría inédita, Universidad Nacional de Mar del Plata, 2014; "La Región Río de la Plata y el comercio ultramarino durante las últimas décadas del siglo XVIII: circulación comercial, mercancías y actores", Tesis de Doctorado inédita, Universidad Nacional de La Plata, 2015.
} 
un mecanismo que denomina centralización corporativa. ${ }^{23}$ Las tensiones locales salen a la luz en 1801, a través de las páginas del Telégrafo Mercantil, en lo que fue el primer debate político que alcanzó la esfera pública a nivel local. El tema: dónde se debían hacer fuertes inversiones para construir un puerto propiamente dicho. ${ }^{24}$

La invasión inglesa de 1806-1807, como sabemos desnudó la incapacidad de la corona de cumplir su parte del pacto de sujeción y sugirió a los intereses vinculados a la producción ganadera y a los servicios de logística que un cambio de política económica sólo podría expandir sus fuentes de ganancias. La opción que se les presentaba era abandonar el "libre comercio" por el "comercio libre". Los comerciantes no estaban muy de acuerdo en esto, tampoco los productores de manufacturas, como los vinculados a los obrajes cochabambinos, o esos zapateros y herreros porteños, cuyas opiniones despreciaba Mariano Moreno por pertenecer a la chusma, en el segundo gran debate político local, el de 1809, que fue el primero de la larga serie de intercambios en torno al proteccionismo y el librecambio.

La guerra que se instala en el río desde 1810, entre porteños y montevideanos en primer lugar, quiebra la unidad regional, y el complejo portuario rioplatense entra en su etapa de crisis y disolución. Las luchas facciosas, sostenidas por diferentes modos de responder a las preguntas planteadas por la caída de la Corona y el rechazo de los poderes que intentan abrogarse la soberanía, abren el camino de la disolución del virreinato y sus intendencias. En el litoral, Santa Fe, Entre Ríos, Corrientes y Montevideo dejan de responder a la capital e inician el camino que llevó a la formación de estados-provincia, que en el caso de la banda norte del río culmina en una república autónoma. En ese litoral, el telón de fondo de las luchas es la realización ultramarina de la riqueza ganadera en expansión, o potencialmente expandible con los saladeros, de economías similares que hasta hacía poco formaban parte de una sola. El proteccionismo es tal vez la gran diferencia con el tempranamente aplastado proyecto artiguista. Me pregunto si, más tarde, a Rosas y a Urquiza el destino de las economías del interior no les interesaba ante todo como mercados para sus socios ultramarinos.

\footnotetext{
${ }^{23}$ Javier Kraselsky, "Los comerciantes rioplatenses y sus estrategias de negociación corporativa. Las Juntas de Comercio en el siglo XVIII", Tesis de Maestría inédita, Universidad Nacional de Tres de Febrero, 2005; "Las estrategias de los actores del Río de la Plata: las Juntas y el Consulado de comercio de Buenos Aires a fines del Antiguo Régimen (17481809)", Tesis de Doctorado inédita, Universidad Nacional de La Plata, 2011.

${ }^{24}$ Telégrafo Mercantil. Rural, político-económico e historiógrafo del Río de la Plata (1801-1802). Reimpresión facsimilar dirigida por la Junta de Historia y Numismática Americana. Tomo VI. Año 1801, Buenos Aires, Compañía Sud-Americana de Billetes de Banco, 1914, V. en los números 3, 4 y 5 los argumentos en favor de Montevideo y en el número 8, 9 y 10 los que proponen la Ensenada de Barragán.
} 
Es distinta la disputa con el interior. Si bien Buenos Aires en los años 1820 creyó que podía desentenderse de su destino, rápidamente tomó conciencia de que su prosperidad dependía de que pudiera seguir presentándose ante el comercio mundial como interlocutora exclusiva de esos mercados. El motivo, siempre el mismo: hay bodegas para sus cueros, a los que ahora se suma la carne salada, en la medida en que hayan llegado cargadas con stocks que su mercado no puede absorber, reducido, además, por los conflictos intrarregionales. Por esos conflictos los porteños también enfrentan un problema serio: la desintegración de la región Río de la Plata en la que llevaban la voz cantante, que traería como derivación la pérdida del control sobre espacios de su campaña, en la banda norte del río y en el sur de Santa Fe y Entre Ríos. De modo que son empujados a otra lucha, hacia el interior y el sur de la pampa, para contar con nuevas tierras en las que poder seguir sustentando su base ganadera, rompiendo la paz establecida con los indígenas por Carlos III sobre la base de renunciar a la continuación de la conquista.

En esta explicación, que mira el siglo XIX desde el XVII y el XVIII, el poder local asociado a los intereses ganaderos no emergería con la revolución y la guerra, sino que es previo y se redefine al calor de las formas que asume en la región la resolución de la crisis general que la lleva a tener que enfrentar un problema no buscado: la desintegración del armado institucional de la dominación. Estaban cómodos como estaban, y por más que se estuvieran cocinando a fuego muy lento en algunas mentes afiebradas ingredientes que podrían haber llevado a la búsqueda de la independencia, en la primera década del siglo XIX, como además ya lo notó un estanciero, Cornelio Saavedra, "las brevas no estaban maduras”, pero no pudieron elegir el momento. ${ }^{25}$

El estado actual de las investigaciones sobre el siglo XIX muestra que para entender la formación del Estado-nación argentino desde los años 1820 en adelante ya no debemos mirar sólo los intereses y la agenda de temas que emergen de Buenos Aires. Los trabajos de Viviana Conti desde la economía salto-jujeña ${ }^{26}$ o un libro coordinado por Beatriz Bragoni y Eduardo Míguez ${ }^{27}$, entre otros muchos esfuerzos, muestran que los estados-provincia del interior no sólo reaccionaban ante Buenos Aires, sino que tenían sus

\footnotetext{
${ }_{25}$ Fernando Jumar, "Los Rioplatenses, el Río de la Plata y el poder central. Hombres de Antiguo Régimen en luchacontra la modernidad", en Karl Kohut; Sonia Rose (Eds.), La formación de la cultura virreinal. III. El Siglo XVIII, Madrid/Frankfurt am Main, Iberoamericana/Vervuert Verlag, 2006, pp. 387-415; "Comercio, comerciantes y revolución en el Río de la Plata. Siglo XVIII - principios del siglo XIX”, en Margarita Guerra Martinière; Cristina Mazzeo de Vivó; Denisse Rouillon Almeida (Eds.), Historias compartidas. Economía, sociedad y poder, siglos XVIXX, Lima, Pontificia Universidad Católica del PerúInstituto Riva-Agüero, 2007, pp. 307-343.

${ }^{26}$ Viviana Conti, “Articulaciones mercantiles del espacio saltojujeño durante el período rosista”, Tesis de Doctorado inédita, Universidad Nacional de La Plata, 2007.

${ }_{27}$ Beatriz Bragoni y Eduardo Míguez (coords.), Un nuevo orden político: Provincias y Estado nacional, 1852-1880, Buenos Aires, Biblos, 2010.
} 
propias agendas. Y vemos con más claridad una vieja explicación del acuerdo final, la dada por Juan Álvarez en torno a que la paz entre las regiones llegó cuando se acordó un proteccionismo selectivo que beneficiaría a las producciones en que sustentaban sus aspiraciones los sectores dominantes regionales. ${ }^{28}$ Se terminó de cerrar un nuevo pacto de dominación, en los términos de Waldo Ansaldi: se soñó con Rousseau y se despertó con Hobbes. ${ }^{29}$ Con ello tomó forma un nuevo espacio económico, que recibe el nombre de mercado interno argentino que desde 1862 organizará el Estado-nación que, por fin, se había logrado crear.

En el tramo final de la lucha (1852-1862), el complejo portuario rioplatense vive sus últimos estertores. Lo veo a través de las explicaciones dadas por Fernando Barba al papel de los derechos diferenciales en la guerra económica entre la Confederación Argentina y el Estado de Buenos Aires, cuando la Confederación intenta redefinir el complejo portuario, cambiando Buenos Aires por sus puertos. ${ }^{30}$ Pero como sabemos, no lo logró y tras Pavón, entre tantas cosas que cambiaron de modo definitivo, el río de la Plata comenzó a ser realmente una frontera internacional.

Así, entiendo, y como se sostuvo al comenzar, que el seguimiento de la vida del complejo portuario rioplatense en la vida regional y en las relaciones con otros espacios regionales americanos y extra-americanos, puede aportar otros ángulos de observación al proceso de formación del Estado-nación argentino, hundiendo raíces en una temporalidad mayor que la señalada por el quiebre de 1810 y dando primacía a los intereses económicos en su traducción política. Los trabajos reunidos en este dossier de modo diverso sostienen los argumentos vertidos, al menos hasta fines del siglo XVIII y comienzos del siguiente. Queda pues, mucho por hacer.

\section{Bibliografía}

Acebal, Maximiliano, "Caminos del río Paraguay: Asunción y sus áreas de influencia económica, 1779-1783”, en 6tas. Jornadas de Historia Económica, Asociación Uruguaya de Historia Económica, Montevideo, 2-4 de diciembre de 2015.

\footnotetext{
${ }^{28}$ Juan Álvarez, Estudio sobre las guerras civiles argentinas, Buenos Aires, Juan Roldán, 1914.

${ }^{29}$ Waldo Ansaldi, "Soñar con Rousseau y despertar con Hobbes: Una introducción al estudio de la formación del Estado nacional argentino”, en Waldo Ansaldi y José Luis Moreno, Estado y Sociedad en el pensamiento nacional, Buenos Aires, Cántaro, 1989, pp. 21-108.

${ }^{30}$ Fernando Enrique Barba, “Algo más sobre el fracaso los derechos diferenciales”, en Investigaciones y Ensayos, 55, 2005, pp. 41-49.
} 
Álvarez, Juan, Estudio sobre las guerras civiles argentinas, Buenos Aires, Juan Roldán, 1914.

Ansaldi, Waldo, "Soñar con Rousseau y despertar con Hobbes: Una introducción al estudio de la formación del Estado nacional argentino”, en Ansaldi, Waldo; Moreno, José Luis, Estado y Sociedad en el pensamiento nacional, Buenos Aires, Cántaro, 1989, pp. 21-108.

Assadourian, Carlos Sempat, "Integración y desintegración regional en el espacio colonial. Un enfoque histórico”, en EURE, Revista Latinoamericana de Estudios Urbanos Regionales, 2, 4, 1972, pp. 11-23.

Assadourian, Carlos Sempat, "Sobre un elemento de la economía colonial: producción y circulación de mercancías en el interior de un conjunto regional”, EURE. Revista Latinoamericana de Estudios Urbanos Regionales, 3, 8, 1973, pp. 135-181.

Barba, Fernando Enrique, "Algo más sobre el fracaso los derechos diferenciales", en Investigaciones y Ensayos, 55, 2005, pp. 41-49.

Biangardi, Nicolás, "Expansión territorial, producción ganadera y relaciones de poder en la región Río de la Plata. Montevideo y Maldonado a fines del siglo XVIII", Tesis de Doctorado inédita, Universidad Nacional de La Plata, 2015, http://www.memoria. fahce.unlp.edu.ar/tesis/te.1179/te.1179.pdf (consulta 11/o2/2019).

Biangardi, Nicolás, "Poblamiento, crecimiento económico y poderes locales en un área de la región Río de la Plata. Maldonado, 1755-1814”, Tesis de Maestría inédita, Universidad Nacional de Tres de Febrero, 2012.

Bragoni, Beatriz; Míguez, Eduardo (Coords.), Un nuevo orden político: Provincias y Estado nacional, 1852-1880, Buenos Aires, Biblos, 2010.

Camarda, Maximiliano, "Comercio Ultramarino de cueros del complejo portuario rioplatense. 1779- 1799”, Tesis de Maestría inédita, Universidad Nacional de Mar del Plata, 2014.

Camarda, Maximiliano, "La Región Río de la Plata y el comercio ultramarino durante las últimas décadas del siglo XVIII: circulación comercial, mercancías y actores", Tesis de Doctorado inédita, Universidad Nacional de La Plata, 2015, http://www.memo- 
ria.fahce.unlp.edu.ar/tesis/te.1181/te.1181.pdf (consulta 11/02/2019).

Conti, Viviana Edith, "Articulaciones mercantiles del espacio saltojujeño durante el período rosista”, Tesis de Doctorado inédita, Universidad Nacional de La Plata, 2007, http://www.memoria.fahce.unlp.edu.ar/tesis/te.228/te.228.pdf (consulta 16/02/2019).

Jumar, "Colonia del Sacramento, la bibliografía y la creación de las memorias nacionales”, en Alejandra Mailhe; Emir Reitano (Comps.), Pensar Portugal. Reflexiones sobre el legado histórico y cultural del mundo luso en Sudamérica, La Plata, Facultad de Humanidades y Ciencias de la Educación, pp. 41-75, http://www.memoria.fahce.unlp. edu.ar/libros/pm.285/pm.285.pdf (consulta 23/05/2018).

Jumar, "Los Rioplatenses, el Río de la Plata y el poder central. Hombres de Antiguo Régimen en lucha contra la modernidad”, en Kohut, Karl; Rose, Sonia (Eds.), La formación de la cultura virreinal. III. El Siglo XVIII, Madrid/Frankfurt am Main, Iberoamericana/Vervuert Verlag, 2006, pp. 387-415.

Jumar, Fernando, “Comercio, comerciantes y revolución en el Río de la Plata. Siglo XVIII - principios del siglo XIX”, en Guerra Martinière, Margarita; Mazzeo de Vivó, Cristina; Rouillon Almeida, Denisse (Eds.), Historias compartidas. Economía, sociedad y poder, siglos XVI-XX, Lima, Pontificia Universidad Católica del PerúInstituto Riva-Agüero, 2007, pp. 307-343.

Jumar, Fernando, "El espacio colonial peruano en la historiografía sobre circulación mercantil", en História Ecônomica \& História de Empresas, 17, 2, 2014, pp. 475534, http://www.abphe.org.br/revista/index.php/rabphe/issue/view/40 (consulta 03/02/2019).

Jumar, Fernando, "El mundo hispánico durante el Antiguo Régimen desde los circuitos mercantiles y el espacio económico rioplatense” en Historia Crítica, 70, Bogotá, 2018, pp. 23-44, https://revistas.uniandes.edu.co/doi/pdf/10.7440/histcrit70.2018.02 (consulta 13/12/2018).

Jumar, Fernando, "El precio de la fidelidad. La Guerra de Sucesión en el Río de la Plata, los intereses locales y el campo Borbón”, en Molinié, Annie; Merle, Alexandra (Dirs.), 
L'Espagne et ses guerres. De la fin de la Reconquête aux guerres de l'Indépendance, París, Presses de l’Université Paris/Sorbonne, 2004, pp. 203-236, http://www. fuentesmemoria.fahce.unlp.edu.ar/libros/pm.9/pm.9.pdf (consulta 05/02/2019).

Jumar, Fernando, "Las 'reformas borbónicas' en la formación de la historiografía argentina” en Historia del Caribe, Universidad del Atlántico, 11, 29, 2016, pp. 69-97, http:// investigaciones.uniatlantico.edu.co/revistas/index.php/Historia_Caribe/article/ view/1533 (consulta 14/11/2018).

Jumar, Fernando, "Les rioplatenses et le Río de la Plata. Les modalités du commerce rioplatense et les groupes locaux, 1680-1777”, Mémoire d'Études Appofondies (=maestría), inédita, École des Hautes Études en Sciences Sociales, 1988.

Jumar, Fernando, Le commerce atlantique au Río de la Plata, 1680-1778, 2da. ed. aumentada, $1^{a}$ ed. electrónica, La Plata, UNLP, 2010, http://www.memoria.fahce. unlp.edu.ar/tesis/te.364/te.364.pdf (consulta 10/02/2019). Publicación de: "Le commerce atlantique au Río de la Plata, 1680-1778”, Tesis de Doctorado, École des Hautes Études en Sciences Sociales, 2000.

Jumar, Fernando. "El complejo portuario rioplatense durante la dominación hispana y su crisis", en Boletín Digital. Academia Nacional de la Historia de la República Argentina, 25, 2016, pp. 30-38, http://anh.org.ar/img/boletines/160/academia-de-la-historia-boletin-digital-25-.pdf (consulta 15/02/2019).

Kraselsky, Javier, "Las estrategias de los actores del Río de la Plata: las Juntas y el Consulado de comercio de Buenos Aires a fines del Antiguo Régimen (1748-1809)", Tesis de Doctorado inédita, Universidad Nacional de La Plata, 2011, http://www.memoria.fahce.unlp.edu.ar/tesis/te.447/te.447.pdf (consulta 09/02/2019).

Kraselsky, Javier, "Los comerciantes rioplatenses y sus estrategias de negociación corporativa. Las Juntas de Comercio en el siglo XVIII", Tesis de Maestría inédita, Universidad Nacional de Tres de Febrero, 2005.

Levene, Ricardo, Las Indias no eran colonias, $1^{\text {a }}$ ed. en 1951, $3^{\text {a }}$ edición, Colección Austral \# 1060, Madrid, Espasa Calpe, 1973. 
Mitre, Bartolomé, Historia de Belgrano y de la Independencia Argentina, $4^{\mathrm{a}}$ ed. definitiva, 3 Tomos, Buenos Aires, Editorial Felix Lajouane, 1887.

Moutoukias, Zacarías, "Le Río de la Plata et l'espace péruvien au XVII ${ }^{\mathrm{e}}$ siècle : commerce et contrebande par Buenos Aires”, Tesis de Doctorado, École des Hautes Études en Sciences Sociales, Francia, 1983. Hay una versión impresa en castellano: Contrabando y control colonial en el siglo XVII, Buenos Aires, CEAL, 1988.

Paredes, Isabel, "El comercio de la subregión de Paraguay a fines del siglo XVIII", en XIII Jornadas Interescuelas/departamentos de Historia, Universidad Nacional del Comahue, San Carlos de Bariloche, 2009, http://cdsa.aacademica.org/ooo-0o8/610. pdf (consulta 10/02/2019).

Paredes, Isabel, “El Sesquicentenario de Mayo, la memoria y la acción editorial”, Universidad Nacional de Tres de Febrero, 2008.

Paredes, Isabel, "La carrera del Paraguay a fines del siglo XVIII”, en América Latina en la Historia Económica, 21, 1, 2014, pp. 66-91.

Paredes, Isabel, "Los transportistas asuncenos de la Carrera del Paraguay y sus relaciones comerciales con Buenos Aires”, en XIV Jornadas Interescuelas/Departamentos de Historia, Universidad Nacional de Cuyo, Mendoza, 2013, https://www.aacademica. org/ooo-010/264 (consulta 10/02/2019).

Pieretti-Bravo, Juan Ignacio, "De comerciante a Apoderado del Gremio de Hacendados. El ascenso político de Antonio Obligado, 1765-1793”, Tesis de Licenciatura inédita, Universidad Nacional de Tres de Febrero, 2018.

Poggi, Marta Mercedes, "Educar al soberano. Los libros escolares, lugares de memoria, 1880-1890". Tesis de Licenciatura inédita, Universidad Nacional de Tres de Febrero, 2004.

Poggi, Marta Mercedes, "La consolidación y difusión de un mito fundacional. La Revolución de Mayo en los textos escolares, 1880-19052, Tesis de Maestría inédita, Universidad Nacional de Tres de Febrero, 2007. 
Sandrín, María Emilia, "El sitio a Colonia del Sacramento, la economía y su gente, 17351737”, Tesis de Maestría, Universidad Nacional de Tres de Febrero, 2011.

Sandrín, María Emilia, "La demanda de bienes y servicios para la Corona y la navegación ultramarina en el complejo portuario rioplatense y la dinamización de la economía regional, 1680-1810", Tesis de Doctorado inédita, Universidad Nacional de La Plata, 2016, http://www.memoria.fahce.unlp.edu.ar/tesis/te.1217/te.1217.pdf (consulta 11/02/2019).

Sandrín, María Emilia, Bizcocheras, lancheros y demás... Los estímulos económicos de la guerra para los sectores medios y bajos del complejo portuario rioplatense, 17351737, Saarbrücken, Editorial Académica Española, 2011.

Serrato Higuera, Rubén Darío, "Las armas del dinero: Mecanismos de financiación y administración en la segunda expedición de Pedro de Cevallos al Río de la Plata, 1777”, Tesis de Maestría, Universidad Nacional de Tres de Febrero, 2017. Hay una versión impresa: Las armas del dinero. El estado fiscal-militar español del siglo XVIII, Saarbrücken, Editorial Académica Española, 2018.

Sovarzo, José, “La garganta del comercio interoceánico en la América del sur hispana. Mendoza y sus relaciones comerciales con Río de la Plata y los mercados del Pacífico Sur americano", Tesis de Maestría inédita, Universidad Nacional de Tres de Febrero, 2017.

Telégrafo Mercantil. Rural, político-económico e historiógrafo del Río de la Plata (18011802). Reimpresión facsimilar dirigida por la Junta de Historia y Numismática Americana. Tomo VI. Año 1801, Buenos Aires, Compañía Sud-Americana de Billetes de Banco, 1914. 